the two formulas was further assessed by comparison with the modified Modification of Diet in Renal Disease (MDRD) equation for Chinese patients, and a composite equation based on the MDRD formula plus cystatin $\mathrm{C}$.

Overall, the model that incorporated both plasma cystatin $\mathrm{C}$ and creatinine concentrations generated estimates of GFR that most accurately reflected true GFR (coefficient of determination $r^{2}=0.83$; median of absolute difference from true GFR $6.89 \mathrm{ml} / \mathrm{min}$ per $1.73 \mathrm{~m}^{2}$ ). Importantly, this combination equation misclassified CKD stage only infrequently, even at near-normal GFRs.

The authors conclude that their final remodeled formula-estimated $\mathrm{GFR}=169$ $\times[\text { creatinine }]^{-0.608} \times[\text { cystatin C }]^{-0.63} \times$ age $^{-0.157}$ ( $\times 0.83$ for females) - could improve estimation of GFR and disease classification in patients with CKD, particularly those with early-stage renal dysfunction.

Original article Ma YC et al. (2007) Improved GFR estimation by combined creatinine and cystatin C measurements. Kidney Int 72: 1535-1542

\section{Colorectal cleansing with oral sodium phosphate is associated with acute kidney injury}

Some studies have found that oral sodium phosphate (OSP) purgatives, which are administered before many of the 14 million lower intestinal endoscopies performed in the US each year, might increase the risks of acute and chronic renal failure in patients with previously normal renal function. Hurst et al. investigated the risk of acute kidney injury (AKI) following OSP use, and compared it with the risk associated with the use of polyethylene glycol (PEG), an alternative purgative agent.

Creatinine values were available for 9,799 patients $(6,432$ of whom had received an OSP purgative, and 3,367 of whom had received a PEG purgative, before an outpatient screening endoscopic procedure). During the 3.4-year study period, serum creatinine levels were measured 87.2 days before and 126.0 days after endoscopy, on average.

$\mathrm{AKI}$, defined as an increase of $\geq 50 \%$ from baseline in serum creatinine concentration, occurred in 114 of 9,799 (1.16\%) patients. Univariate analysis detected a nonsignificantly increased risk of AKI in the OSP group (1.29\% vs $0.92 \%$ of patients; odds ratio $1.41,95 \%$ Cl 0.93-2.13; $P=0.113)$. Multivariate analyses, which adjusted for factors such as age, diabetes, hypertension and congestive heart failure, found OSP purgatives to be associated with a significantly increased risk of $\mathrm{AKI}$ (odds ratio $2.35,95 \% \mathrm{Cl} 1.51-3.66 ; P<0.001)$. The authors suggest that older patients and those with comorbidities should be given PEG rather than OSP for pre-endoscopy bowel cleansing, and that those who receive OSP should be monitored closely after the procedure.

Original article Hurst FP et al. (2007) Association of oral sodium phosphate purgative use with acute kidney injury. J Am Soc Nephrol 18: 3192-3198

\section{Influenza vaccination does not increase the relapse rate in ANCA-associated vasculitis}

In a retrospective study, 230 consecutive patients with antineutrophil cytoplasmic antibody (ANCA)-associated vasculitis who were followed up for at least 1 year were identified from the outpatient records of a tertiary referral center in The Netherlands. All patients provided information on their influenza vaccination status from October 1999 to December 2003 (about 60\% were vaccinated each year), and data on all relapses during the period October 1999 to December 2004 were obtained from patient records.

Overall, 166 relapses occurred during the study period; 78 were in patients who had been vaccinated in the year before relapse, and 88 were in patients who had not (relapse rates per 100 at-risk patients were 3.4 and 6.3, respectively). Diseasefree survival for each year from 1999 to 2003 was higher in patients who had been vaccinated than in patients who had not, with this difference being statistically significant in 2001 and 2002 . Few differences were noted between the disease and patient characteristics of those participants vaccinated at least once and those who had never been vaccinated, although the mean age and mean duration of disease before enrollment were both greater in vaccinated patients.

The authors conclude that vaccination against influenza does not increase disease activity in patients with ANCA-associated vasculitis.

Original article Stassen PM et al. (2007) Influenza vaccination does not result in an increase in relapses in patients with ANCA-associated vasculitis. Nephrol Dial Transplant [doi:10.1093/ndt/gfm640] 